\title{
Research on Power System Transient Stability Assessment Based on Statistical Learning Theory
}

\author{
Wanyu Xu \\ Department of Electrical Engineering,North China Electric Power University,Baoding, China \\ 757968298@qq.com
}

Keywords: Transient stability assessment; Bagging; Support vector machine; Data set reconstruction

\begin{abstract}
This paper presents a method of model construction for the power system transient stability assessment based on statistical learning theory integrated with the bagging and the approximate reasoning. Support vector machines operate on the principle of structure risk minimization. This paper takes full advantage of its ability to solve the problem with small sample, nonlinear and high dimension. Hence better generalization ability is guaranteed. The multi-class identification for power system transient stability assessment is solved by the data set reconstruction. The assessment model uses the data set regulation, bagging and approximate reasoning to improve the training speed, the accuracy and stability of the estimation result. The IEEE 39-Bus test system is employed to demonstrate the validity of the proposed approach.
\end{abstract}

\section{Introduction}

In recent years, the application of artificial intelligence technology in power system transient stability assessment made great progress, in which the use of artificial neural networks to find the mapping between the system parameters and the steady state performance indexes was the most active. But these methods practical applications still exist some problems, mainly as follows: (1) The generalization error of transient stability assessment can not be guaranteed. (2) the model parameter selection is difficult and training results are not stable. To solve the above problems, this paper uses support vector machine developed from statistical learning theory to build a new transient stability assessment model[1]. The proposed model contains bagging and approximate reasoning strategy, which can make accurate reasoning for the unknown sample and improves the stability and accuracy of the prediction.

\section{Support vector machine}

The support vector machine (SVM) developed from statistical learning theory can effectively solve high-dimensional, nonlinear pattern recognition problem. Through the non-linear transformation, the input vector is mapped to a high-dimensional space $H$. Construct optimal hyperplane in $H$, and achieve the best generalization ability[2].

Set a sample data set $E=\left(x_{1}, y_{1}\right), \cdots,\left(x_{p}, y_{p}\right)$, where $x_{i} \in R^{n}$ is input vector, which describe the system condition; $y_{i}$ is output vector, which describe system operating mode; $p$ is the number of samples; $n$ is the dimension of input vector.

According to SVM, the input vector can be mapped to a high feature space by nonlinear transformation $K\left(x, x_{i}\right)$ satisfied Mercer condition, which is shown in Fig.1. Then, build the best 
classification Hyperplane $f(x)$ in the proposed feature space:

$$
f(x)=\operatorname{sign}\left(\sum_{i=1}^{p} y_{i} \alpha_{i} K\left(x, x_{i}\right)+b\right)
$$

Where sign is sign function; the nonlinear transformation $K\left(x, x_{i}\right)$ is called kernel function; $\alpha_{i}$ is Lagrange multipliers; $b$ is the offset.

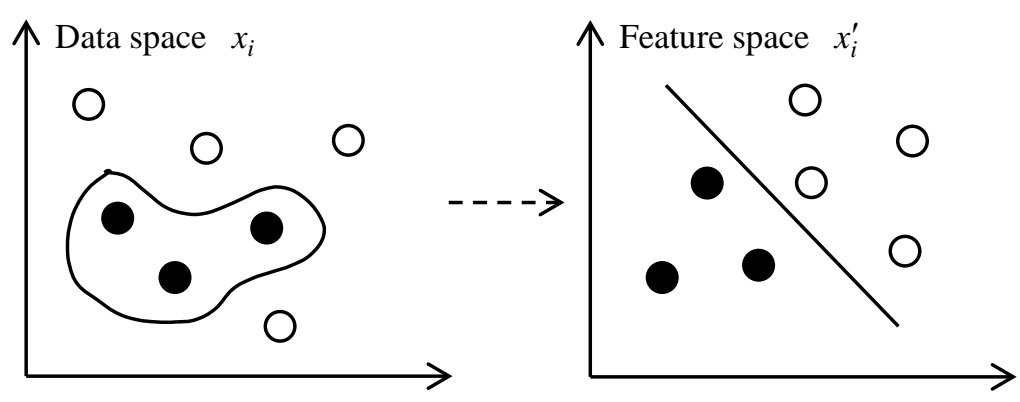

Fig. 1 Mapping from data space to feature space

$\alpha_{i}$ can be obtained from the following programming:

$$
\begin{gathered}
\min \Omega=\frac{1}{2} \sum_{i=1}^{p} \sum_{j=1}^{p} \alpha_{i} \alpha_{j} y_{i} y_{j} K\left(x_{i}, x_{j}\right)-\sum_{i=1}^{p} \alpha_{i} \\
\text { s.t } \quad \alpha_{i} \geq 0 \quad i=1,2, \cdots, p \\
\sum_{i=1}^{p} \alpha_{i} y_{i}=0
\end{gathered}
$$

According to Karush-Kuhn-Tucker (KKT) condition, the samples which satisfy $\alpha_{i} \neq 0$ are the "critical samples” which affect the classification result, called support vector.

\section{The construction of transient stability assessment model}

This model mainly consists input and output modules, input standardized modules, training sample output reconstruction module, transient stability assessment sub-planar configuration module and temporarily stable approximate reasoning machine modules. Each module functions are described as follows:

(1) The input and output of transient stability assessment

After a lot of simulation, this paper constructs the original sample set of transient stability assessment $E_{S}=\left(x_{1}, y_{1}\right), \cdots,\left(x_{o}, y_{o}\right)$, shown in Fig.2, where $x_{i} \in R^{18}$ is a 18-dimension feature vector describing the features of power system, shown in Table.1; $y_{i} \in R$ is 1-dimension vector indicating the system transient stability degree; $o$ is the number of samples[3]. 
Table.1 Input feature of data set

\begin{tabular}{|c|c|c|c|}
\hline No. & Input feature vector & No. & Input feature vector \\
\hline x1 & Total system load & x10 & $\begin{array}{c}\text { The total system generating } \\
\text { capacity }\end{array}$ \\
\hline x2 & Part nodes load & x11 & Part bus voltage \\
\hline x3 & Average system bus voltage & x12 & The Max-min value of bus voltage \\
\hline x4 & Fault location & x13 & Excitation gain \\
\hline x5 & $\begin{array}{c}\text { Generator failure instant } \\
\text { acceleration }\end{array}$ & x14 & $\begin{array}{c}\text { Failure instant generator } \\
\text { acceleration power }\end{array}$ \\
\hline x6 & $\begin{array}{c}\text { The maximum acceleration } \\
\text { generator initial angular }\end{array}$ & $\mathrm{x} 15$ & COI fault instant acceleration \\
\hline x7 & $\begin{array}{c}\text { Cut the instantaneous generator } \\
\text { angle }\end{array}$ & $\mathrm{x} 16$ & $\begin{array}{c}\text { Cut the instantaneous generator } \\
\text { acceleration }\end{array}$ \\
\hline $\mathrm{x} 9$ & $\begin{array}{c}\text { Fault instantaneous generator } \\
\text { kinetic energy }\end{array}$ & $\mathrm{x} 17$ & $\begin{array}{c}\text { Removal generator kinetic } \\
\text { moment }\end{array}$ \\
\hline & $\begin{array}{c}\text { COI removal instantaneous phase } \\
\text { angle change }\end{array}$ & $\mathrm{x} 18$ & Total system energy adjustment \\
\hline
\end{tabular}

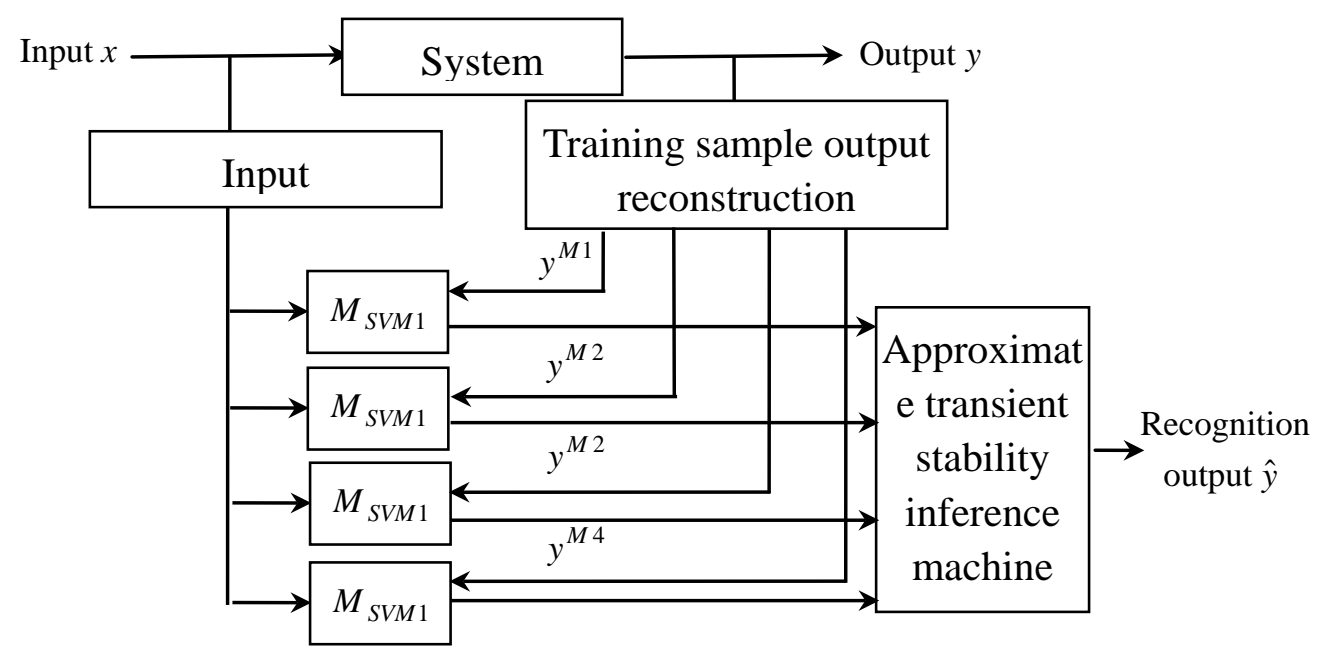

Fig. 3 Model construction for transient stability assessment

According to the difference of limit clearing time $T_{c c t}$ of different samples, the range of one-dimensional vector $y_{i}$ is defined as $\{1,2,3,4\}$. Where 1 indicates that the system is unstable; 2 represents the system is marginally stable; 3 indicates the system is stable; 4 indicates that the system is very stable.

(2) Input standardization and training sample output reconstruction

In this paper, zero-mean normalization is used to transfer the original sample set, and form the original normalization data set:

$$
d^{\prime}=(d-\bar{L}) / \sigma_{L}
$$

Where $\bar{L}$ and $\sigma_{L}$ are respectively the average and standard deviation of $L ; d^{\prime}$ is the standardization value of $d$ belonging to $L$.

Then, randomly select a certain percentage of the original normalization data as training 
samples $E_{\text {train }}$. The other samples are regarded as testing sample $E_{\text {test }}$. Through the reconstruction for the output of $E_{\text {train }}$, the training sample of $M_{S V M i}$ are obtained, shown in Fig.3. The reconstruction process should obey the following condition:

$$
\left\{\begin{array}{l}
y_{k}^{M i}=1, \quad \text { if } y_{k}=i, \forall y_{k} \in E_{\text {train }} \\
y_{k}^{M i}=-1, \quad \text { if } y_{k} \neq i, \forall y_{k} \in E_{\text {train }}
\end{array}\right.
$$

(3) The construction of transient stability assessment sub-plane

According to SVM theory, this paper uses Gaussian radial basis function $K\left(x, x_{i}\right)$ to construct transient stability assessment sub-plane $f_{i}$.

$$
f_{i}(x)=\operatorname{sign}\left(\sum_{i=1}^{p t} y_{i} \alpha_{i} K\left(x, x_{i}\right)+b\right)
$$

Where $p t$ is the size of the training set. The Gaussian radial basis function is shown as follows:

$$
K\left(x, x_{i}\right)=\exp \left(-\frac{\left(x-x_{i}\right)^{2}}{2 \sigma^{2}}\right)
$$

\section{The improvement of transient stability assessment model}

This paper construct the corresponding bagging model $M_{\text {Baggingi }}$ to replace the single transient stability assessment sub-plane $f_{i}$. Here, take the replace process of $M_{S V M_{i}}$ as an example. Use the boot method to pick out the transient stability training subset $S_{j}(j=1, \cdots, T)$ of the training set $M_{S V M_{i}}$. Then, use SVM to build the transient stability prediction model $V_{j}(j=1, \cdots, T)$. Combining the $T$ transient stability prediction models can form the bagging model $M_{\text {Baggingi }}$ [4].

Putting the unknown stability degree sample $U$ into $M_{\text {Bagging1 }}$ can judge the stability of $U$ according to the prediction value of each SVM model $V_{j}(j=1, \cdots, T)$.

$$
\sum_{j \in\{1, \cdots, T\}}\left(V_{j}=1\right)>\sum_{j \in\{1, \cdots, T\}}\left(V_{j}=-1\right)
$$

Where the left represents all the SVM prediction models whose output is 1, and the right represents all the SVM prediction models whose output is -1. If the Eq.(7) is satisfied, the output result of $M_{\text {Bagging1 }}$ is 1 , indicating that $U$ belongs to the stability level of $M_{\text {Bagging } 1}$. In contrast, if the output result of $M_{\text {Bagging1 }}$ is -1 , it means that $U$ does not belong to the stability level of $M_{\text {Bagging1 }}$. Following the same steps, the paper can obtain the final composition transient stability assessment 
model consisting of four bagging models[5].

\section{Case study}

This paper takes IEEE 39-Bus node test system as example. Randomly select fault type and location, obtain a total of 2000 samples. Select the former1400 samples as training sample, the remaining 600 samples as testing samples. Each bagging model is consist of three SVM models. Enter the testing sample and test the bagging model $M_{\text {Bagging } 1}$. The testing results are shown in Table.2. (Due to limited space, only lists eight samples)

Table. 2 Test of $M_{\text {Bagging1 }}$ vote machine

\begin{tabular}{|c|c|c|c|c|c|}
\hline $\begin{array}{c}\text { Testing } \\
\text { sample }\end{array}$ & $\begin{array}{c}\text { Actual } \\
\text { stability }\end{array}$ & $\mathrm{V} 1$ & $\mathrm{~V} 2$ & $\mathrm{~V} 3$ & $\begin{array}{c}M_{\text {Bagging1 }} \\
\text { prediction result }\end{array}$ \\
\hline 1 & 4 & -1 & -1 & -1 & -1 \\
\hline 2 & 1 & 1 & 1 & 1 & 1 \\
\hline 3 & 1 & 1 & -1 & 1 & 1 \\
\hline 4 & 3 & -1 & -1 & -1 & -1 \\
\hline 5 & 2 & -1 & -1 & -1 & -1 \\
\hline 6 & 2 & -1 & -1 & -1 & -1 \\
\hline 7 & 1 & 1 & 1 & -1 & 1 \\
\hline 8 & 2 & 1 & 1 & 1 & 1 \\
\hline
\end{tabular}

As can be seen from Table.2, through the voting $M_{\text {Bagging1 }}$, No.3 sample is judged correctly,

which not only improves the transient stability prediction accuracy, but also makes the results more stable classification. However, No.8 sample makes a wrong judgment, which requires to get the final result by a further approximate reasoning. The testing results of the final composition of the transient stability assessment model are shown in Table. 3.

Table. 3 Result judged by predicted model

\begin{tabular}{|c|c|c|}
\hline Sample set & The number of misjudged sample & Correct rate [\%] \\
\hline Training sample set & 11 & 99.2 \\
\hline Testing sample set & 24 & 96.0 \\
\hline
\end{tabular}

\section{Conclusions}

Through constructing support vector machine, this paper find the optimal division hyperplane and boundary samples which judges the transient stability of power system. This model uses the data set regulation, bagging and approximate reasoning to improve the training speed, the accuracy and stability of the estimation result. Application results in IEEE 39-Bus test system proves the effectiveness of this method of power system transient stability monitoring, which provides a new way of thinking for solving the power system transient stability assessment.

\section{References}

[1] Aboytes F, Ramirez R. Transient stability assessment in longitudunal power system using artificial neural networks. IEEE Transaction on Power system. Vol. 11 (1996), p. 2003-2010. 
[2] Vapnik V. The natural of statistical theory. New York: Springer-Verlag, 1995.

[3] Nello C, John S T. An introduction to support vector machines and other kernel-based learning methods. Cambridge: Cambridge University Press, 2000.

[4] Hsu Ch W, Lin Ch J. A comparison of methods for multi2 class support vector machines. IEEE Transactions on Neural Networks. Vol. 13 (2002), p. 415-425.

[5] Ding M, Huang K, Li SH. Probabilistic stability assessment for hybrid AD/DC power systems. Proceeding of the CSEE. Vol. 22 (2002), p. 11-16. 